\title{
Utilização da técnica de Difração de Raios-X para o estudo de amostras de Ti-6Al-4V forjadas e fabricadas por Fusão Seletiva a Laser
}

Use of the X-ray Diffraction technique for the study of samples of Ti-6Al-4V forged and manufactured by Selective Laser Melting

Max Amorim Brum¹, Fábio Beck², Oreci Escobar da Silva³, Gustavo Luiz Callegari,

Marcos André Carara ${ }^{5}$, Sabrina Marques ${ }^{6}$ e Josué Neroti Rigue ${ }^{7}$.

1,2,3,6,7 Universidade Federal de Santa Maria- Campus Cachoeira do Sul.

maxbrum1991@gmail.com; fabiobeckster@gmail.com; josue.rigue@ufsm.br; josue.rigue@ufsm.br; josue.rigue@ufsm.br

${ }^{4}$ Departamento de Física - Universidade Federal de Santa Maria

glcallegari@gmail.com; josue.rigue@ufsm.br

\section{Resumo}

A busca por materiais com características bem definidas é de fundamental importância para qualquer projeto de engenharia. Além disso, sabe-se que os vários tipos de processos de fabricação influenciam diretamente nas propriedades das peças. Neste trabalho foi utilizada a técnica de difração de raios-X para estudar as características estruturais de amostras de Ti6Al-4V fabricadas pelas técnicas de forjamento e Fusão Seletiva a Laser. Percebeu-se que as amostras fabricadas por Fusão Seletiva a Laser chegaram a apresentar uma redução de $1 \%$ no parâmetro de rede perpendicular ao plano estudado, quando comparadas com as amostras forjadas. Como a maioria das peças fabricadas por FSL atende a demandas de projetos nas áreas aeronáutica e aeroespacial, onde tensões residuais geralmente são indesejáveis por tornar as peças mais frágeis, estudos como esse adquirem grande relevância.

Palavras-chave: Difração de raios-x; Estrutura cristalina; Lei de bragg; Fusão seletiva a laser; Indústria 4.0; Manufatura avançada

\section{Abstract}

The search for materials with well-defined characteristics is very important for any engineering project. In addition, it is known that the various types of manufacturing processes directly influence in properties of the parts. In this work, the X-ray diffraction technique was used to study the structural characteristics of Ti-6Al-4V samples manufactured by the forging and Selective Laser Melting techniques. We found that the samples made by FSL were able to present a reduction of $1 \%$ in the lattice parameter perpendicular to the studied plane, when compared with the forged samples. Since most of the parts manufactured by Selective Laser Melting meet the demands of aeronautical and aerospace projects, where residual stresses are generally undesirable for making the parts more fragile, studies like these acquire great relevance.

Keywords: X-ray difraction; Cristalline structure; Bragg's law; Selective laser melting; 4.0 Industry; Advanced manufacturing 


\section{Introdução}

Com os avanços tecnológicos cada vez mais acelerados, impulsionados pela nova era industrial, processos de manufatura avançada e a proximidade da $4^{\mathrm{a}}$ Revolução Industrial (Indústria 4.0), novas técnicas de fabricação mais rápidas e precisas vem se destacando nesse ambiente. O processo de Fusão Seletiva a Laser (FSL) trata-se de um desses processos inovadores e tecnológicos, que tem por objetivo fabricar peças complexas com as mesmas características mecânicas das fabricadas por métodos mais tradicionais. O objetivo desse trabalho é realizar uma comparação entre as propriedades de corpos de prova fabricados por FSL e pelo método de forjamento tradicional através da técnica de difração de raios-X.

\section{Referencial teórico}

A proposta desse trabalho é utilizar a técnica de difração de raios-X que, entre outras utilidades, serve para identificar fatores referentes à estrutura atômica dos materiais, para estudar amostras produzidas por dois importantes métodos. Para isso, cabe uma breve revisão sobre os temas envolvidos.

Os raios-X, assim como a luz, são ondas eletromagnéticas e foram descobertos em 1895 pelo físico alemão Wilhelm Conrad Röntgen e receberam esse nome devido a sua natureza desconhecida na época. Segundo Cullity (1956), esses raios são invisíveis, viajam em linhas retas e afetam filmes fotográficos da mesma forma que a luz. Todavia, eles são mais penetrantes e podem facilmente passar pelo corpo humano, madeira, pedaços grossos de metal e outros objetos "opacos".

O método mais utilizado para produzir raios-X é fazendo com que um elétron de alta energia gerado em um cátodo colida com um alvo metálico (ânodo). Quando esse elétron atinge o alvo, pode ocorrer que um elétron da camada K de um dos átomos do material seja liberado, fazendo com que haja uma vacância nessa camada. Para ocupar o espaço deixado por esse elétron, um outro elétron de uma camada mais externa passa a ocupar essa vacância na camada K, liberando energia na forma de Raio-X (BLEICHER \& SASAKI, 2000).

Cullity (1956) também salienta que, embora a radiografia seja uma ferramenta muito importante e tenha um vasto campo de aplicabilidade, é normalmente limitada no detalhamento interno, para tamanhos da ordem de $10^{-1} \mathrm{~cm}$. A difração, por outro lado, pode indiretamente revelar detalhes de estrutura da ordem de $10^{-8} \mathrm{~cm}$.

Segundo o mesmo autor, a difração deve-se essencialmente à existência de certas relações de fase entre duas ou mais ondas. Os raios-X podem ser absorvidos e difratados pelos cristais do material que está sendo analisado e percorrem caminhos diferentes ao longo da estrutura do material, com distâncias diferentes. Isso pode fazer com que as ondas entrem em fase ou não. A figura 1 abaixo apresenta o caminho seguido pela radiação no interior de um material. A relação que associa a saída em fase dos feixes difratados (condição de interferência construtiva) com a distância percorrida pela radiação no interior do material, ao comprimento de onda e ao ângulo de incidência radiação é a chamada Lei de Bragg, a qual está destacada na figura 1. 
Figura 1 - Ilustração da difração dos raios-X mostrando os parâmetros associados com a Lei de Bragg.

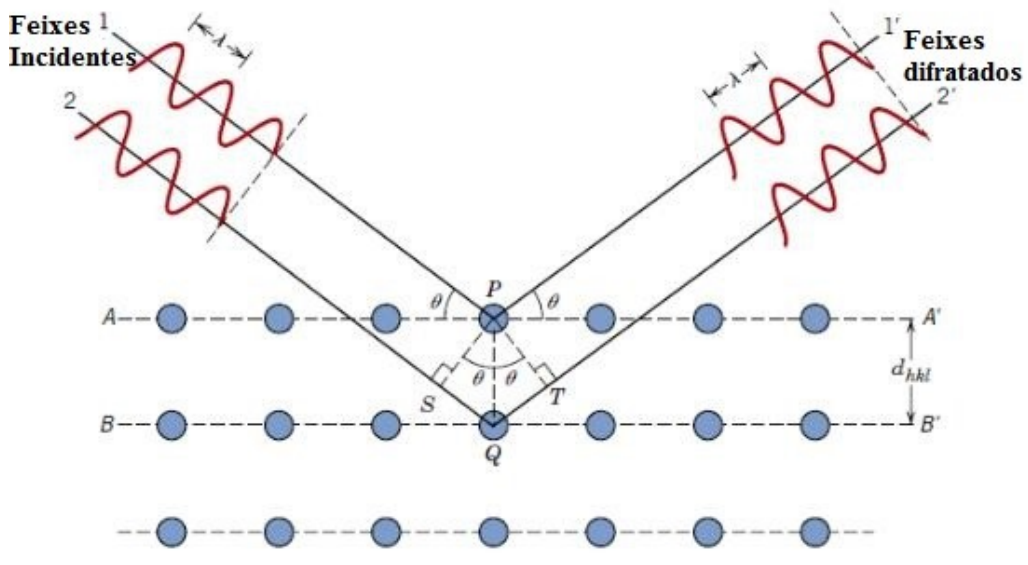

$n \lambda=2 d_{h k l} \operatorname{sen} \theta$

(Lei de Bragg)

O dispositivo utilizado para esse tipo de medida, mostrado na figura 2(a), chama-se difratômetro de raio-X. Como indicado na figura, os raio-X são gerados no tubo de raio-X e direcionados para a amostra que se quer estudar e, após sofrerem a difração no material, são medidos no detector. Quando os feixes difratados encontram-se em fase, picos de intensidades são captados pelo detector, gerando gráficos característicos de acordo com a composição da amostra. Esses gráficos, chamados de difratogramas (figura 2(b)) podem oferecer informações relevantes sobre o material analisado, como rugosidade, densidade, tipo de estrutura cristalina e a composição.

Figura 2 - (a) Difratômetro Bruker D8 Advance disponível no laboratório de magnetismo e materiais magnéticos (LMMM) da UFSM; (b) Difratograma gerado pelo software de gerenciamento do dispositivo.

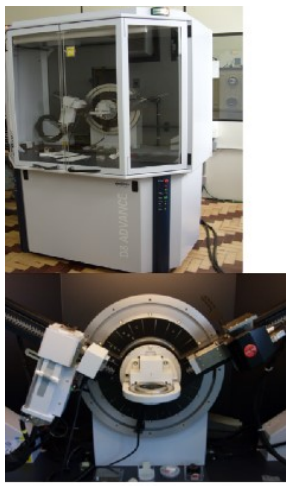

(a)

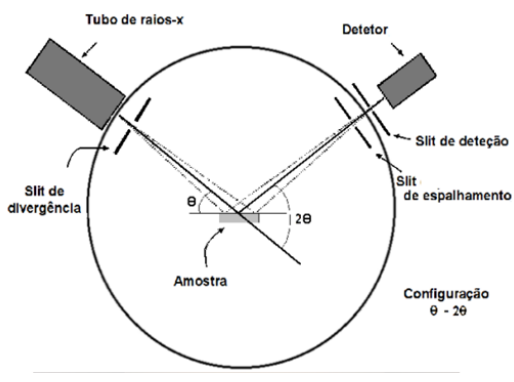

(b)

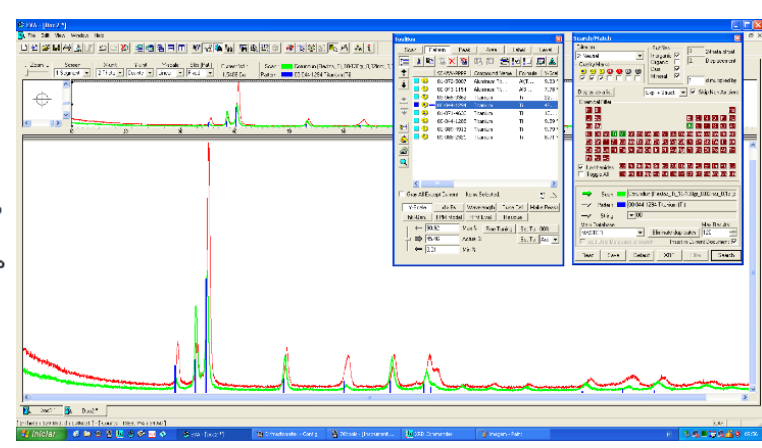

Para compreendermos como os raios-X se comportam ao se moverem pelos planos interatômicos, também é válida uma análise sobre os tipos de estruturas cristalinas conhecidas. Os materiais são analisados de acordo com as características que as estruturas cristalinas demonstram. As propriedades físicas, químicas e mecânicas dos materiais são diretamente influenciadas pela organização atômica que os mesmos apresentam. De acordo com Heczko (2006), um cristal ideal é construído pela repetição de uma unidade estrutural idêntica no espaço. A menor unidade estrutural pode compreender muitos átomos ou moléculas. Em todas as amostras analisadas, a estrutura cristalina encontrada foi do tipo 
hexagonal compacta (HCP). Para maior entendimento sobre os tipos de estruturas cristalinas conhecidas recomenda-se a leitura do livro de Callister Jr, que encontra-se referenciado neste trabalho.

Visando a fabricação de peças com densidade muito próximas a $100 \%$, que seria a ausência de espaços vazios no interior da peça oriundos da fundição da matéria-prima que é pó, que iniciaram-se estudos sobre o processo de FSL. Além disso, esse processo permite a fabricação de peças com geometrias complexas. Ele consiste, basicamente, no varrimento de um feixe de laser sobre a superfície de uma fina camada de pó previamente depositada sobre um substrato. Cada camada da peça é sequencialmente preenchida com filetes alongados de pó fundido, ou seja, peças fabricadas por FSL são a sobreposição de filetes e camadas. Uma vez que os objetos 3D em FSL são construídos camada por camada, a morfologia da camada determina as propriedades do produto final (MARQUES, SOUZA e ZANATTA, 2014).

$\mathrm{O}$ tradicional processo de forjamento, por sua vez, é um processo que consiste na conformação mecânica no qual utiliza forças de compressão para moldar o metal na forma desejada, com ou sem o auxílio de uma matriz, produz peças que geralmente necessitam de longos ciclos de pós-processamento. A FSL tem o objetivo de produzir peças com as mesmas características dos processos de conformação mecânica, porém sem a necessidade de pós-processamentos ou, ao menos, com ciclos bem menores.

Ambos os processos interferem diretamente nas características apresentadas pelas peças produzidas. Este trabalho busca realizar uma análise de tensões residuais nas peças fabricadas por esses dois processos utilizando a técnica de difração de raios-X. Sabe-se que tensão residual geralmente é uma característica indesejada, podendo causar fragilidade nas peças fabricadas e ocasionar o seu colapso antes de atingir a tensão de ruptura característica do material avaliado.

\section{Metodologia}

Para fins de comparação entre os dois tipos de amostras produzidas, os testes foram feitos em corpos de prova constituídos de Ti-6Al-4V produzidos tanto por forjamento, quanto por FSL. Esta liga é bastante utilizada na indústria aeronáutica, aeroespacial e médica. Informações mais completas podem ser encontradas no link disponível nas referências.

Os resultados obtidos para as amostras são mostrados na figura 4 abaixo, onde podemos identificar o resultado para a amostra forjada pela cor preta e a produzida por FSL na cor vermelha. Os difratogramas na figura 4, confirmam a predominância do titânio na composição das amostras e, além disso, indicam a formação da estrutura hexagonal compacta (HC) para o titânio. Os picos de intensidade que surgem nos difratogramas experimentais nos permitem comparar com fichas catalográficas disponíveis na literatura (linhas verdes indicadas na figura), onde é possível encontrar os difratogramas referentes a diferentes estruturas de crescimento para todos os elementos da tabela periódica. Como é possível perceber na região ampliada da figura 4, em vários ângulos existe um deslocamento nos picos para a amostra produzida por FSL, esse deslocamento está associado a uma diferença nos parâmetros de rede para essa amostra, mais precisamente nos parâmetros de redes perpendiculares ao plano em que o feixe de raio-X foi incidido. Utilizando a Lei de Bragg encontramos que nas amostras produzidas por FSL ocorreu, em certas direções, uma redução de aproximadamente $1 \%$ na distância entre os planos atômicos, em comparação com as forjadas. 
Figura 4 - Difratograma gerado através da análise das amostras de Ti-6Al-4V.

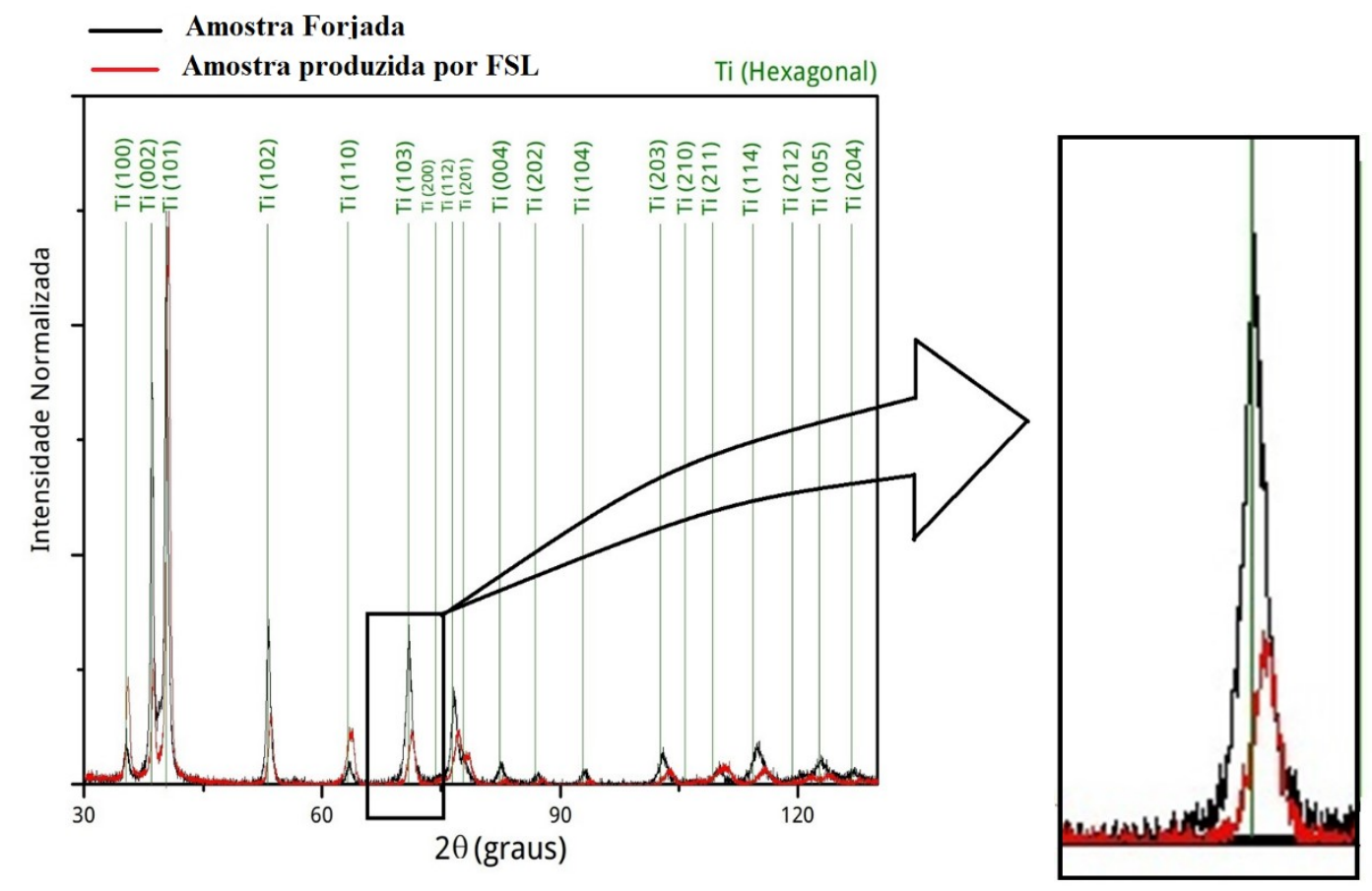

\section{Conclusões}

A análise em ambas as amostras revelou unicamente a presença da estrutura cristalina do tipo hexagonal compacta (HCP) do Titânio e também concluiu-se que houve uma redução de aproximadamente $1 \%$ na distância entre os planos atômicos para as amostras fabricadas pelo processo de FSL, em comparação com as amostras forjadas.

A tensão residual geralmente é um fator indesejado e pode deixar o material frágil, o que ocasiona o seu colapso antes de atingir a tensão de ruptura característica. Assim sendo, é necessária uma avaliação detalhada da situação antes de avançar em um projeto e, também, a continuação dos estudos a respeito do processo de fabricação por FSL para minimizar esse efeito. Ainda, estudos podem ser realizados para averiguar os efeitos de tratamentos térmicos nesses parâmetros que foram avaliados.

\section{Agradecimentos}

Trabalho apoiado pelo programa de Auxílio à pesquisa de recém-doutores (FIPE-Júnior/UFSM).

\section{Referências}

BLEICHER, L., SASAKI, J.M. Introdução à difração de raios-x em cristais. Fortaleza: Universidade Federal do Ceará, 2000.

CALLISTER JR, W.D. Fundamentals of Material Science an Engineering. $5^{\mathrm{a}}$ ed. New York: John Willey \& Sons, 2001. 
CULLITY B. D., Elements of X-ray diffraction (Addison-Wesley, Reading, 1956).

HECZKO, O. X-Ray Diffraction and Structure Analysis Introduction. Teknillisen fysiikan ohjelmatyö, 2006. Disponível em: http://people.sabanciuniv.edu/burc/SU\%20web\%20material_files/MAT\%20509\%20Material/crystal\%20structure/Xraylab200 6.pdf.

MARQUES, S.; SOUZA, A.F.; ZANATTA, A. M. Fusão seletiva a laser para fabricação de peças metálicas com geometrias complexas. SETIS- III Seminário de Tecnologia Inovação e Sustentabilidade, 2014. Disponível em:

http://www4.fiescnet.com.br/images/institutos/artigo2014\%20SETIS\%20FSL.pdf

Ti6Al4V: Titanium Alloy Arcam EB System. Disponível em: http://www.arcam.com/wp-content/uploads/Arcam-Ti6Al4VTitanium-Alloy.pdf . 